\title{
Generalized regression neural network for prediction of peak outflow from dam breach
}

\begin{abstract}
Several techniques have been used for estimation of peak outflow from breach when dam failure occurs. This study proposes using a generalized regression artificial neural network (GRNN) model as a new technique for peak outflow from the dam breach estimation and compare the results of GRNN with the results of the existing methods. Six models have been built using different dam and reservoir characteristics, including depth, volume of water in the reservoir at the time of failure, the dam height and the storage capacity of the reservoir. To get the best results from GRNN model, optimized for smoothing control factor values has been done and found to be ranged from 0.03 to 0.10 . Also, different scenarios for dividing data were considered for model training and testing. The recommended scenario used $90 \%$ and $10 \%$ of the total data for training and testing, respectively, and this scenario shows good performance for peak outflow prediction compared to other studied scenarios. GRNN models were assessed using three statistical indices: Mean Relative Error (MRE), Root Mean Square Error (RMSE) and Nash - Sutcliffe Efficiency (NSE). The results indicate that MRE could be reduced by using GRNN models from $20 \%$ to more than $85 \%$ compared with the existing empirical methods.
\end{abstract}

Keyword: Dam safety; Dam failure; Breach outflow; Peak outflow discharge; Generalized regression neural network 